\title{
Doeg als Incubant.
}

\section{Zur Erklärung von I Sam. 2I, 8.}

Von Erwin Preuschen in Darmstadt.

Bei der an sich klaren und lebendigen Schilderung von David's Flucht aus Gibea bedarf die erste Einführung des Edomiters Doeg der Erklärung, die ihr bisher nur unvollkommen zu Teil geworden ist. Über die Situation im Ganzen kann kein Zweifel bestehen. Sauls Eifersucht ist nach den kriegerischen Erfolgen Davids so starkgeworden, dass er ihn definitiv zu beseitigen trachtet (I Sam. I9, IO). Da flieht David

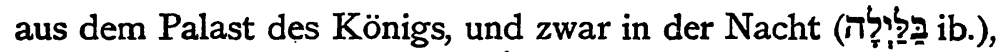
und begiebt sich in sein eignes Haus. Michal kennt die Stimmung ihres Vaters, und da sie David lieb hat $(18,20)$, warnt sie ihn: "wenn du dich nicht in dieser Nacht noch rettest, wirst du morgen sterben." (I9, II) Nun setzt sie mit weiblicher List seine Flucht ins Werk, lässt ihn durch ein Fenster hinab, und er konnte so entrinnen. Die Flucht ist nur möglich, wenn sie durch die Nacht gedeckt ist, sonst hatte ja die ganze Manipulation keinen Sinn. In derselben Nacht flieht David zu Ahimelech, dem Priester von Nob.

Hier beginnt nun die Schwierigkeit, weil man nicht weiss, wo Nob zu suchen ist. In Bêt-Nûbâ darf man es nicht suchen, denn David's Flucht ging nicht nach Westen, sondern nach Süden, seiner Heimat entgegen. Südlich von Gibea liegt aber kein Ort, den man ungezwungen mit Nob identi- 
fizieren könnte. Wenn man an Scha' fât, etwa $2 \mathrm{~km}$. nördlich von Jerusalem gedacht hat (Baedeker, Syrien u. Palästina 3 S. 242), so würde die Lage wohl passen, aber der Name bleibt unerklärt. Cheyne (Encycl. Bibl. III, 3430)

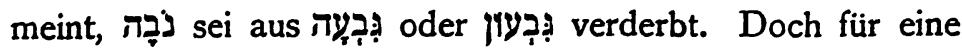
solche Radikalkur fehlen die Grundlagen. Die Frage mag auf sich beruhen. Aus dem Verlauf der Erzählung geht deutlich hervor, dass David noch in der Nacht oder in den ersten Morgenstunden zu Ahimelech gekommen sein muss, und dass folglich Nob nicht allzuweit von Gibea gelegen haben kann. Das ergiebt sich aus dem Umstand, dass noch kein frisches Brot gebacken ist $(21,5)$. Brot wird täglich gebacken, da die dünnen Kuchen zu sehr austrocknen (Winer, Bibl. Real. Wörterbuch I, I29 u. Anm. I). Ahimelech hat aber noch kein frisch gebackenes Brot zur Hand und kann darum David nur von dem geheiligten Brote anbieten. Er muss sich daher erst erkundigen, ob David kultisch rein ist (2I, 5). Als ihm das bejaht wird, giebt er David ohne Bedenken die Schaubrote. Die Motivierung in V. $7^{\mathrm{b}}$,,denn es war dort kein Brot ausser den Schaubroten, die weggenommen waren, damit frischgebacknes Brot aufgelegt würde am Tage, an dem man es wegnahm", kommt nach dem vorhergehenden unerwartet. Es scheint als sollte damit Ahimelech gerechtfertigt werden, dass er David von dem Schaubrote gab. Es war schon weggenommen, da an diesem Tage frisches aufgelegt werden sollte; so war es im Grunde, scheint V. $7^{\mathrm{b}}$ zu meinen, kein heiliges Brot mehr, und Ahimelech beging eben keine allzugrosse Sünde, es wegzugeben. Nach dem Vorhergehenden erwartet man gerade das Gegenteil. Und erst, wenn neues Brot vorhanden ist, scheint es Zeit, das alte wegzunehmen. Daher scheint V. $7^{\text {b eine Glosse }}$ zu sein, die den Zusammenhang unterbricht und stört.

Um das heilige Brot zu holen, muss Ahimelech in den 
Tempel gehen. Dort aber ist Doeg, der Edomiter, der den Vorgang belauscht und Saul Meldung macht von der Richtung, in der David geflohen ist und von der Unterstützung, die er bei Ahimelech gefunden hat. Wie kommt Doeg in den Tempel und was hat er dort getrieben? Die Frage wird in V. 8 beantwortet, aber die Antwort ist undeutlich geworden; und man hat sie daher nicht verstanden. Der Text lautet,

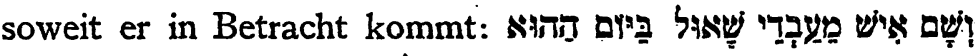

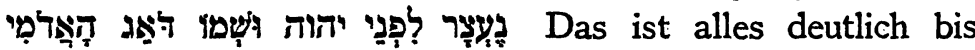

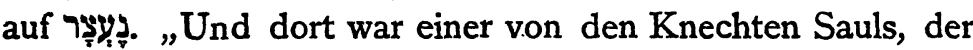
sich hatte einschliessen lassen vor Jahwe, und sein Name war Doeg, der Edomiter.“. Was soll es heissen, dass er „sich hatte einschliessen lassen vor Jahwe"? „Vor Jahwe“ kann nach dem Zusammenhang nur auf das Tempelhaus gehen. Abzuweisen ist weiter jeder Erklärungsversuch, der in der Richtung verläuft, für עצ deutung einzuschieben, als die gewöhnliche. Auf diesem Wege ist schon das Targum des Jonathan vorangegangen,

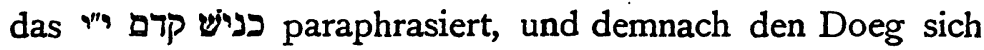
vor Jahwe „versammeln lässt"“. Wie er das anfing, ist dunkel. Noch dunkler, wie David so verwirrt sein konnte, bei der Flucht, die er heimlich $z \mathfrak{u}$ halten allen Grund hatte, in eine Festversammlung. hineinzulaufen, ganz abgesehen davon, dass man in der Nacht doch keine Feste zu feiern pflegt.

Die gewöhnliche Deutung geht darauf hinaus, dass Doeg sich an dem Heiligtum aufgehalten habe, um sich von irgend einer kultischen Unreinheit $\mathrm{zu}$ lösen. So, wie es scheint, der Syrer, bei dem es heisst: 1.0. ح. H. P. Smith, A critical and exegetical Commentary on the Books of Samuel S. 200 erklärt: as the root is used above for that which is religiously forbidden (taboo), we may suspect that it means here, kept by a taboo, or in accordance with later custom, kept by a 
vow, (so Schm. who compares the law of the Nazirite, Num. 6 , but this does not require a sejourn in the sanctuary). Keine dieser Erklärungen befriedigt. ${ }^{x}$ Die erste, die auf W.R.Smith, Rel. of the Semites ${ }^{2} 455$, Deutsche Übersetzung

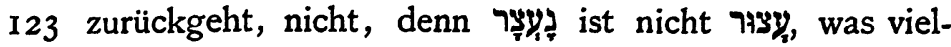
leicht I Kö. 14, 10. Jer. 36, 5. Neh. 6, 10 in der von W. R. Smith angenommenen Bedeutung steht. Ein Unreiner hat nichts im Heiligthum zu suchen, und auch der ny nach Jer. 36, 5 nicht. Wenn der Prophet Schema'ja, trotzdem er עצ ist, Nehemia verleiten will, sich mit ihm im Tempelhaus einzuschliessen, so liegt wahrscheinlich auch darin eine dem Nehemia gestellte Falle. Ist der Unreine wieder rein, so . kann er wieder das Heiligtum betreten, bis dahin hat er es zu meiden. Ebensowenig kann man aber an ein Gelübde

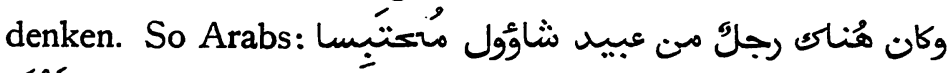

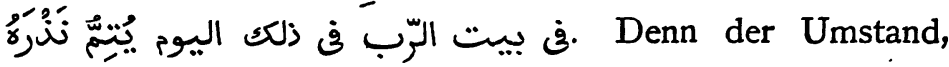
dass das Gelübde dahin ging, sich im Tempel einschliessen zu lassen, wäre so singulärer Natur gewesen, dass er zu erwähnen gewesen wäre. Am bequemsten hat es sich die Vulgata gemacht, wenn sie schlankweg übersetzt: Erat autem ibi vir quidam de servis Saul in die illa intus in tabernaculo Domini. Damit ist die Frage, was denn Doeg

I Bei der Correctur gehen mir die beiden neuen deutschen Kommentare zu Samuelis von K. Budde (Kurzer Hand-Commentar Lief. I8) und W. Nowack (Handkommentar I, 4) zu. Nowack registriert mit einem ,vielleicht“ die erste dieser Erklärungen. Budde : „Er war unter Klausur im Gotteshause, das heisst wohl durch ein Gelübde, eine Busse, ein rituelles Verfahren vorübergehend an das Gotteshaus gebunden, wohl auch die Nacht hindurch" fügt eine Möglichkeit (Busse) hinzu, gegen die dieselben Gründe wie gegen Smith's zweite Erklärung geltend zu machen sind. Statt Budde's „wohl auch die Nacht hindurch" müsste es nach der Situation heissen ,und zwar gerade während der verflossenen Nacht"s. 
im Tempel trieb, dem Scharfsinn des Lesers zur Beantwortung preisgegeben.

Auszugehen ist bei jedem Erklärungsversuch von der Grundbedeutung von كَ, zurückhalten, hindern; darausentwickeln sich die weiteren Bedeutungen, festhalten, einsperren u. ä. von selbst. Dieser Bedeutung zufolge muss das Niph. übersetzt werden: „sich festhalten lassen" oder „sich einschliessen lassen“. Diesem Thatbestande wird auch die Übersetzung der LXX gerecht, bei der allerdings jetzt nicht. mehr alles glatt ist. Sie

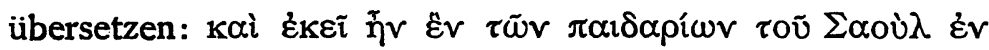

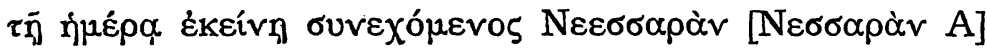

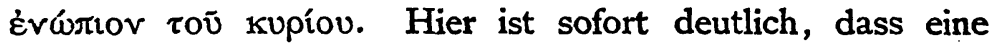
Dublette vorliegt: zunächst die Transcription des Wortes

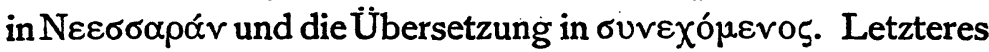
kann uns allein interessieren. Es giebt das Wort genau wieder, aber es enthält auch einen Hinweis auf die Erklärung. Dabei ist es gleichgültig, ob die Glosse бuvexópevos erst

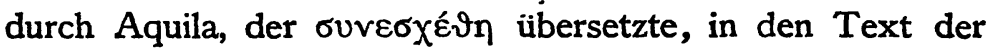
LXX hineingekommen ist, oder nicht. Noch wichtiger aber

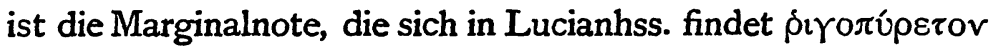

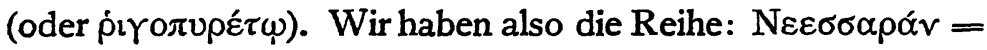

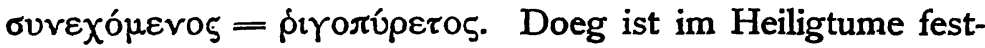
gehalten und zwar, wie die Marginalnote meint, als ein im Fieberschauer liegender Mann.

Damit ist das Rätsel gelöst. Wir können nun ahnen, was den Mann nach dem Tempel trieb, und warum er sich nächtlicher Weile dort im Heiligtume aufhielt. Er war erkrankt und suchte in einem Traumorakel Hilfe gegen seine Krankheit. Der Brauch, im Heiligtum zu schlafen, um auf diese Weise einen Weg zur Gesundheit zu finden, ist bei den Griechen etwas ganz gewöhnliches gewesen. Die grosse Krankheitsgeschichte, die der Rhetor Aelius Aristides 
erzählt, ist bekannt. Seitdem man die Inschriften von dem Asklepiustempel in Epidaurus kennt, hat man dafür inschriftliche Belege in Fülle. Auch im Orient kennt man die Sitte. Als Alexander der Gr. schon im Sterben lag, liess man bei Serapis noch ein Traumorakel befragen. Auch sonst sind wir über die bei der Incubation geübten Gebräuche ausreichend unterrichtet (vgl. die Zusammenstellung bei Deubner, de incubatione p. I4 sqq).

Es ist interessant, dass man diese Sitte auch an den israelitischen Kultstätten übte. Dass man Traumorakel in

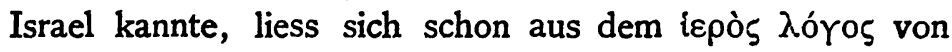
Bethel Gen. 28, II f. und aus I Kö 3 erschliessen. Dass aber auch das medizinische Traumorakel - vorausgesetzt dass die Glosse Lucians Recht hat, - in Übung war, ist neu und bemerkenswert. Es illustriert aufs neue den Sinn des Satzes, dass Gott es den Seinen im Schlafe giebt. 